\title{
Medical Errors Disclosure: Is It Good or Bad?
}

\author{
Amirashkan Nasiripour ${ }^{1 *}$, Pouran Raiessi ${ }^{2}$, Mehrnoosh Jafari ${ }^{1}$ \\ ${ }^{1}$ Department of Health Services Management, Science and Research Branch, Islamic Azad University, Tehran, Iran \\ ${ }^{2}$ Research Department, School of Management and Medical Information Sciences, Iran University of Medical Sciences, \\ Tehran, Iran
}

*Corresponding Author: Amirashkan Nasiripour, Ph.D., Associate Professor, Department of Health Services Management, Science and Research Branch, Islamic Azad University, Tehran, Iran. Tel: 98-9121991372,

Email: nasiripour@srbiau.ac.ir

Received November 9, 2017; Accepted January 17, 2018; Online Published February 4, 2018

\begin{abstract}
Background: In the treatment and health process, there are a lot of dangers to patients, and the increased number of medical errors is one of the most important circumstances of this process.

Objective: The present research purposed to decrease medical errors through disclosure of them in hospitals of Tehran University of Medical Sciences.

Methods: This qualitative-quantitative study included a population of 232 individuals, selected through targeted non-random selection, who were somehow connected with medical errors made in hospitals affiliated with Tehran University of Medical Sciences. The triangulation technique was utilized to collect data. Data was analyzed using the factor analysis technique. After the effective factors were determined, their internal correlation was determined through regression correlation.

Results: Disclosure is made up of organizational culture, the factors related to the patient, the factors related to the presenter, the factors involved in the error, structural factors, and disclosure situation indexes, and is an effective factor in managing the medical errors in hospitals of Tehran University of Medical Sciences. Disclosure increases the immunity of patients.

Conclusion: Disclosure is an effective factor in managing the medical errors that occur at hospitals affiliated with Tehran University of Medical Sciences. It is essential that managers identify the effective factors in disclosure and take steps to apply a suitable disclosure system to decrease errors.

Keywords: Disclosure, Hospitals, Medical Errors
\end{abstract}

\section{Background}

The issue of patient safety has obtained a special place in presenting treatment and health services. An increasing number of patients all over the globe are hurt every year through errors which are caused by healthcare, and adverse events have occurred as the effect of these errors. Different actions have been taken with regard to patient safety, and progress has been made in line with establishing a safer health system. Still with these advances, the errors remain unavoidable, and they happen in the area of medicine as well as other complicated systems. ${ }^{1}$

External and internal statistics exist on the occurrence of medical errors, and they double the necessity for attending to the problem and support the importance of the issue:

Human and financial loads caused by medical errors in the health system were calculated on the basis of two large studies, one conducted in 1984 and the other in 1992 in the United States, in which the rate of medical errors among all hospitalized patients were respectively calculated to be $2.9 \%$ and $3.7 \%$, respectively. Addition, taking into account all hospitalized patients in the hospital, respectively $58 \%$ and $53 \%$ of all adverse events that occurred during treatment processes could have been caused by medical errors which could have been avoided. ${ }^{2}$

InEuropean countries, onein every 6 doctors is prosecuted because of medical violations. The number of prosecutions for medical violations in Europe is continuously increasing in a way that it has caused defensive medicine to become a global phenomenon in Europe. ${ }^{3}$

Research carried out on errors in using medication committed by 60 nursing students in the coronary care unit of Arak indicated that medication errors occurred in $10 \%$ of the units under research; 41.66 reported medication errors which were about to happen, and $48.34 \%$ of the samples did not report any errors or errors which were about to happen. ${ }^{4}$

Taking into account the above- mentioned statistics, the occurrence of medical errors and the consequential adverse events have become an unavoidable characteristic of treatment and health systems all around the world. In

Copyright (C) 2018 The Author(s). This is an open-access article distributed under the terms of the Creative Commons Attribution License (http:// creativecommons.org/licenses/by/4.0), which permits unrestricted use, distribution, and reproduction in any medium, provided the original work is properly cited. 
Iran, therefore, identifying, preventing, and effectively managing these errors and preventing their repeated occurrence have become major priorities in treatment and health systems. ${ }^{2}$

Fundamental step to managing medical errors are to discover, identify, and report medical errors; it has been shown by different studies that, unless an error is reported and registered, it cannot be managed. ${ }^{5}$

The effective management of medical errors has presented error disclosure as an important strategy for decreasing errors. ${ }^{6-10}$ Put in a better way, informing people accurately and rightfully about the issues related to patient safety and the processes which must be in reach, having an open and mutual relationship with patients and their companions about errors that occur, expressing regret for the event, and making an effort to compensate for it effectively are important indexes in managing medical errors disclosure which, of course, have an impressive role in decreasing reoccurrence and the number of medical errors. ${ }^{11}$

According to medical ethics, when errors occur, they should be revealed and expressed honestly. According to paragraphs 8 and 12 of the Medical Ethics Law of the Medical Association of the United States, for more than 20 years this association has been committed to saving all patients from all wrong decisions which endanger their health conditions and inform them of errors if they occur. ${ }^{12}$

\section{Objective}

The major aim of this research was to decrease medical errors through studying the role of disclosure (expressing the error in a friendly environment between the presenter and the patient) and reporting (admitting the error has occurred and expressing it in an official and administrative way to the hospital) in hospitals of Tehran University of Medical Sciences.

\section{Methods}

This mixed (qualitative and quantitative) research used the triangulation method (review of literature, interview, and questionnaire).

The population of the research included all individuals who participated in executing medical activities in hospitals of Tehran University of Medical Sciences between the years 2009-2010. There are 26 hospitals affiliated with the named university. Four of them were excluded from this study because they did not want to participate. A team of 13 people was selected through purposive sampling consisting of the chief of the hospital, the manager of the hospital, the director of quality improvement, the nurse manager, the chief of the intensive case section, the chief of one of the clinical sections, the chief of the surgery room and anesthesia section, the clinical supervisor, the educational supervisor, the head nurse of the section, the director of the pharmacy, the doctor who was the director of the emergency section, and the chief of the clinical governance office. The total sample being studied consisted of 13 individuals from 22 hospitals, or 286 individuals. Fifty-four questionnaires were not returned; therefore, the sample consisted of 232 people, and taking into account the fact that 22 individuals (from each hospital along with a nurse from the emergency section) were also interviewed the total number of the sample reached 254 individuals.

Three instruments (receipt card, interview, and questionnaire) were used to collect data. At first, texts and literature related to medical errors were studied, and the effective components on disclosure of medical errors were extracted. ${ }^{7,8,13-32}$ Based on the extracted components, the interview questions were developed. Non-structured interviews were conducted with 20 experts from clinical governance offices. The interviews consisted of the following four questions: (1) How much hospital culture is effective in the occurrence of errors, and what cultural factors do you see more effective in making errors? (2) What features in patients make medical errors easier for hospital managers? (3) Which factors in the hospital structure, such as manpower management, focus, high work load, reward and punishment, and similar issues in managing errors, are effective in medical errors management? How does each factor work? (4) Which characteristics of providers (doctors, nurses, etc) are important in handling errors? Finally, a researcher-made questionnaire was established using the information obtained from the review of the texts and the interview. The questionnaire included 2 parts, demographic information and information related to the variables under study, and it consisted of 47 questions. First the content and face validity of the questionnaire was determined through expert judgment. The questionnaire was distributed to ten experts (four experienced researchers in the field of medical and patient safety errors, four mentors and counselors, a statistical adviser, and a Ph.D. in Persian literature who edited the writing). The face validity was determined with the help of a pilot implementation. Then, after the field research, the questionnaire was presented to the respondents in two forms, Internet-based and in person. All the collected data from the questionnaire was analyzed using SPSS version 22 software (through factor analysis, regression analysis, and correlation).

\section{Results}

After the validity was approved, the reliability of the questionnaire was calculated to be 0.98 through Cronbach a. The results of the factor analysis test indicated that the data was suitable for factor analysis. This result approved the results of both capabilities of the data tests for carrying out factor analysis taking into account the significant amounts of $(\mathrm{kmo}=0.892)$ and also the Bartlett test (3634.609) on a $P<0.001$ level.

It was determined that error disclosure is comprised of organizational culture, the factors related to the presenter, the factors related to the patient, the factors related to error, disclosure situation, structural factors, and reporting the errors consisting of individual factors and 
structural factors. The mentioned factors express $47 \%$ of the variance of data, of which the bigger share comprises the organizational culture factor, and the smaller share comprises the organizational factors affecting reporting (Table 1). Table 2 shows the factor comprising error management and its comprising variables.

The results of regression analyses and correlation indicated that there is a relationship between error disclosure and managing the errors. $t$ criteria and its constituent factors are larger than 1.96, and the level of significance is smaller than 0.05 , which justifies the existence of the relationship. There was a relationship between managing the errors and error disclosure, and the results indicated that there is a correlation between these two indexes (Table 3).

After the effective factors and the comprising indexes of managing medical error were determined through regression analysis and route analysis, the final model of the research and the capability of the model to express the indexes and effective factors were presented (Figure 1 and Table 4).

\section{Discussion}

Error disclosure is one of the most important factors in managing and controlling medical errors in the hospitals of Tehran University of Medical Sciences. The results of the current study regarding the effect of this factor on managing errors was along the lines of those of the studies of Leape, ${ }^{20}$ Etchegaray et al, ${ }^{21}$ Ghalandarpoorattar et al, ${ }^{22}$ Kronman et al ${ }^{23}$ Greene, ${ }^{24}$ Vozikis, ${ }^{25}$ Levinson, ${ }^{26}$ Waite, ${ }^{27}$ Moskop et al, ${ }^{28}$ Chan et al, ${ }^{29}$ Fein et al, ${ }^{30}$ Kalra, ${ }^{31}$ Nekoei. ${ }^{32}$ Of course, the generalizability of the factors affecting disclosure, which were studied in the results of this study, were not in all of the mentioned studies. Differences in a number of the mentioned factors could be the reason for the differences in the study environment, in the naming of the factors, or the opinion of the researcher in randomly or purposefully selecting the effective factors.
Table 1. Organizational Factors Affecting Reporting

\begin{tabular}{lccc}
\hline \multirow{2}{*}{ Factors } & \multicolumn{3}{c}{ Rotation Sums of Squared Loadings } \\
\cline { 2 - 4 } & Total & Variance & Cumulative Variance \\
\hline 1 & 3.893 & 9.054 & 9.054 \\
2 & 3.380 & 7.859 & 16.913 \\
3 & 2.743 & 6.378 & 23.291 \\
4 & 2.664 & 6.196 & 29.487 \\
5 & 2.451 & 5.700 & 35.187 \\
6 & 2.101 & 4.887 & 40.074 \\
7 & 1.825 & 4.245 & 44.319 \\
8 & 1.498 & 3.483 & 47.802 \\
\hline
\end{tabular}

As is shown in the results of this and similar studies, hospitals must fundamentally take notice of error disclosure which is the main demand of the patient, agreed upon and approved by the professional and committed organizations. This is increasingly and progressively necessary for the governmental and legal body of the hospitals. The benefits of this affair are directed at the presenter of the services, the receiver of the services, and the system in which errors occur as well.

Reporting errors is not an effective factor in managing them. The findings of this study regarding the effects of this factor on error management differ from those of Fischer et al, ${ }^{33}$ Hartnell et al, ${ }^{34}$ Moumtzoglou, ${ }^{35}$ Vozikis, ${ }^{25}$ Hosford, ${ }^{36}$ Hoover, ${ }^{37}$ Walsh and Antony, ${ }^{38}$ Thomas and Petersen, ${ }^{39}$ Uribe et $\mathrm{al},{ }^{40}$ and Harenstam et al. ${ }^{41}$

\section{Conclusion}

Since the possibility of errors occurring is a part of natural life and humans are constantly prone to a vast range of errors, the occurrence of these errors is an unavoidable affair in treatment and healthcare systems. However, due to the greater sensitivity and the even vaster range of consequences as a result of errors in healthcare systems, controlling and managing errors in healthcare systems is of more importance and is more essential in comparison

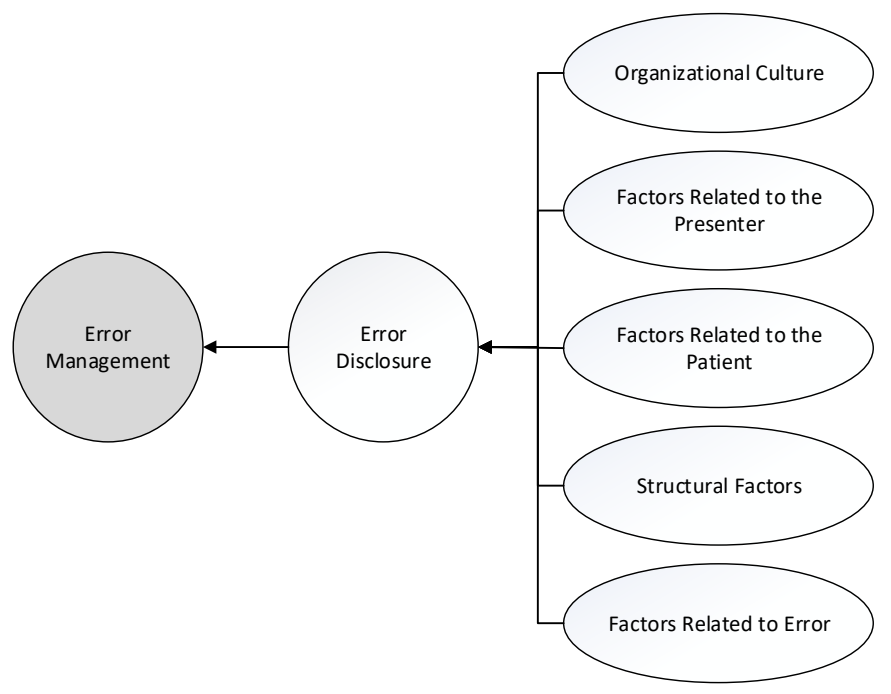

Figure 1. Managing Errors and the Factors and Indexes Comprising it. 


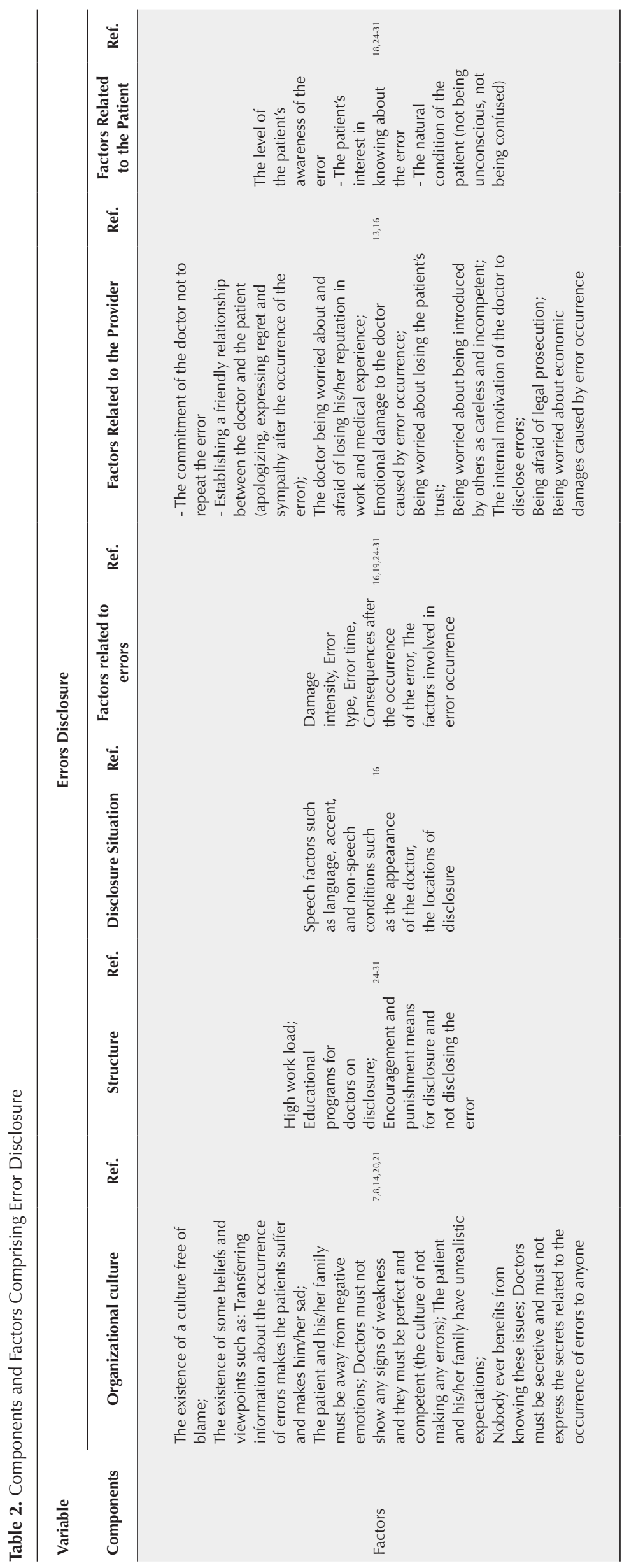


Table 3. Regression of Managing Medical Errors and the Factors Comprising it

\begin{tabular}{|c|c|c|c|c|c|}
\hline & \multicolumn{2}{|c|}{ Non-standardized Coefficient } & \multirow{2}{*}{$\frac{\text { Standardized Coefficient }}{\text { B }}$} & \multirow{2}{*}{$t$} & \multirow{2}{*}{$P$ Value } \\
\hline & $\boldsymbol{\beta}$ & Standard Error & & & \\
\hline Managing medical errors (constant variable) & 0.952 & 0.708 & & 1.343 & 0.181 \\
\hline Error disclosure & 0.305 & 0.017 & 0.737 & 18.285 & 0.000 \\
\hline
\end{tabular}

Table 4. Effects of Direct and Indirect Independent Variables of the Study on the Dependent Variable (Error Management)

\begin{tabular}{|c|c|c|}
\hline Type of the Effect & Route & The Amount of the Effects on the Basis of Standardized Coefficients \\
\hline Direct & Error disclosure & 0.737 \\
\hline Sum of direct effects & & 0.737 \\
\hline \multirow{8}{*}{ Indirect } & Organizational culture & 0.310 \\
\hline & Factors related to the presenter & 0.280 \\
\hline & Factors related to the patient & 0.283 \\
\hline & Factors related to the error & 0.303 \\
\hline & Disclosure situation & 0.117 \\
\hline & Structural factors & -0.053 \\
\hline & Individual factors & 0.606 \\
\hline & Organizational factors & 0.162 \\
\hline Sum of indirect effects & & 2.008 \\
\hline Sum of direct and indirect factors & & 2.745 \\
\hline
\end{tabular}

with other systems. Revealing errors to the patient through a mutual relationship along with honesty, compassion, and kindness, supporting the patient, and supporting the presenter with the viewpoint that anyone can make mistakes have very important positions in the controlling cycle.

In line with making this a reality, it is necessary for a transition to take place in the traditional viewpoint of human errors in the hospitals of Iran. For this transition to take place, it is necessary for cultural backgrounds, such as studying the error as a multifaceted event and one that has various causes, not punishing and blaming people, accepting that to err is human, emphasizing the promotion of professional relationships and teamwork, to exist. It is worth mentioning that creating one such environment requires a set of decisions and measures and the development of basic and practical studies around this topic in the future.

\section{Research Highlights}

\section{What Is Already Known?}

Research on medical errors in Iran is rare. More studies have looked at the magnitude of errors, not their causes or ways to control them.

\section{What This Study Adds?}

This article examines the disclosure of medical errors and, with a comprehensive review of the literature on this research, addresses all the factors affecting disclosure. Disclosure as a very effective and important factor in reducing and controlling errors.

\section{Authors' Contributions}

Study design: AN, PR; Data collection: MJ, AN; Data analysis: AN, PR, MJ; and manuscript preparation: MJ.

\section{Conflict of Interest Disclosures}

The authors declare that they have no conflicts of interest.

\section{Ethical Approval}

Not applicable.

\section{Acknowledgments}

The authors would like to thank all the managers of hospitals affiliated with Tehran University of Medical Sciences for their valuable contributions. It should be noted that this article is a substitute for a doctoral dissertation in the Department of Health Services of Science and Research Branch of Islamic Azad University, Tehran.

\section{References}

1. Yardley IE, Yardley SJ, Wu AW. How to discuss errors and adverse events with cancer patients. Curr Oncol Rep. 2010;12(4):253260. doi:10.1007/s11912-010-0109-0.

2. Dabagh A, Akbari ME, Fathi M. Medical errors patterns in health care. Iran Islamic Republic Army Journal. 2006;4:957966.

3. Inelmen EM, Sergi G, Enzi G, et al. On clinical errors in geriatric medicine diagnosis: ethical issues and policy Implication. Ethics Med. 2010;26:15-24.

4. Mardani Hamole M, Shahraki Vahed A. Barriers of Medical errors reporting in nurses. Ethics Med Hist. 2009;2:56-60.

5. Cao H, Stetson $\mathrm{P}$, Hripcsak G. Assessing explicit error reporting in the narrative electronic medical record using keyword searching. J Biomed Inform. 2003;36(1-2):99-105. 
doi:10.1016/S1532-0464(03)00058-3.

6. Kolaitis IN, Schinasi DA, Ross LF. Should medical errors be disclosed to pediatric patients? Pediatricians' attitudes toward error disclosure. Acad Pediatr. 2016;16(5):482-488. doi:10.1016/j.acap.2015.06.011.

7. Youngson GG. Medical error and disclosure - a view from the U.K. Surgeon. 2014;12(2):68-72. doi:10.1016/j. surge.2013.10.011.

8. Kachalia A, Bates DW. Disclosing medical errors: the view from the USA. Surgeon. 2014;12(2):64-67. doi:10.1016/j. surge.2013.12.002.

9. Hannawa AF, Shigemoto Y, Little TD. Medical errors: Disclosure styles, interpersonal forgiveness, and outcomes. Soc Sci Med. 2016;156:29-38. doi:10.1016/j.socscimed.2016.03.026.

10. Heaton HA, Campbell RL, Thompson KM, Sadosty AT. In support of the medical apology: the nonlegal arguments. J Emerg Med. 2016;51(5):605-609.doi:10.1016/j.jemermed.2016.06.048.

11. Disclosure of medical errors. Ann Emerg Med. 2004;43(3):432. doi:10.1016/j.annemergmed.2003.11.014.

12. Ghajarzade M. Medical errors: doctors guide. https://www. tums.ac.ir/. Published 2009. [Persian].

13. Tsiga E, Panagopoulou E, Montgomery A. Examining the link between burnout and medical error: A checklist approach. Burn Res. 2017;6:1-8. doi:10.1016/j.burn.2017.02.002.

14. Tevlin R, Doherty E, Traynor O. Improving disclosure and management of medical error - an opportunity to transform the surgeons of tomorrow. Surgeon. 2013;11(6):338-343. doi:10.1016/j.surge.2013.07.008

15. Kachalia A, Bates DW. Disclosing medical errors: the view from the USA. Surgeon. 2014;12(2):64-67. doi:10.1016/j. surge.2013.12.002

16. Abd Elwahab S, Doherty E. What about doctors? The impact of medical errors. Surgeon. 2014;12(6):297-300. doi:10.1016/j. surge.2014.06.004

17. Hannawa AF. Disclosing medical errors to patients: effects of nonverbal involvement. Patient Educ Couns. 2014;94(3):310313. doi:10.1016/j.pec.2013.11.007.

18. Leone D, Lamiani G, Vegni E, Larson S, Roter DL. Error disclosure and family members' reactions: does the type of error really matter? Patient Educ Couns. 2015;98(4):446-452. doi:10.1016/j.pec.2014.12.011.

19. Giraldo P, Castells X. [Medical errors and the apology laws, do we need them?]. Med Clin (Barc). 2015;145(8):341-343. doi:10.1016/j.medcli.2015.04.019.

20. Leape LL. Apology for errors: whose responsibility? Front Health Serv Manage. 2012;28(3):3-12. doi:10.1097/01974520201201000-00002.

21. Etchegaray JM, Gallagher TH, Bell SK, Dunlap B, Thomas EJ. Error disclosure: a new domain for safety culture assessment. BMJ Qual Saf. 2012;21(7):594-599. doi:10.1136/ bmjqs-2011-000530.

22. Ghalandarpoorattar SM, Kaviani A, Asghari F. Medical error disclosure: the gap between attitude and practice. Postgrad Med J. 2012;88(1037):130-133. doi:10.1136/ postgradmedj-2011-130118.

23. Kronman AC, Paasche-Orlow M, Orlander JD. Factors associated with disclosure of medical errors by housestaff. BMJ Qual Saf. 2012;21(4):271-278. doi:10.1136/bmjqs-2011-000084.

24. Greene D. Nurses Experience with the Disclosure of Errors to Patient. College of Health and Human Science: Georgia State
University; 2009.

25. Vozikis A. Information management of medical errors in Greece: The MERIS proposal. Int J Inf Manage. 2009;29(1):1526. doi:10.1016/j.ijinfomgt.2008.04.012.

26. Levinson W. Disclosing medical errors to patients: a challenge for health care professionals and institutions. Patient Educ Couns. 2009;76(3):296-299. doi:10.1016/j.pec.2009.07.018.

27. Waite MA. Patient Safety and Disclosure of Medical Error: The Legal and Ethical Implications of Human Error in Medicine. Masters Abstracts. 2006;44:2152.

28. Moskop JC, Geiderman JM, Hobgood CD, Larkin GL. Emergency physicians and disclosure of medical errors. Ann Emerg Med. 2006;48(5):523-531. doi:10.1016/j. annemergmed.2006.04.007

29. Chan DK, Gallagher TH, Reznick R, Levinson W. How surgeons disclose medical errors to patients: a study using standardized patients. Surgery. 2005;138(5):851-858. doi:10.1016/j. surg.2005.04.015.

30. Fein S, Hilborne L, Kagawa-Singer $\mathrm{M}$, et al. A conceptual model for disclosure of medical errors. Advances in Patient Safety. 2003;2:483-494

31. Kalra J. Medical errors: overcoming the challenges Clin Biochem. 2004;37(12):1063-1071. doi:10.1016/j. clinbiochem.2004.08.008.

32. Nekoei A. Nurse's working shift work fatigue and errors. Summarized Articles of the Second National Conference on Prevention of Medical Errors; 2007;141.

33. Fischer MA, Mazor KM, Baril J, Alper E, DeMarco D, Pugnaire $M$. Learning from mistakes. Factors that influence how students and residents learn from medical errors. J Gen Intern Med. 2006;21(5):419-423. doi:10.1111/j.1525-1497.2006.00420.x

34. Hartnell N, MacKinnon N, Sketris I, Fleming M. Identifying, understanding and overcoming barriers to medication error reporting in hospitals: a focus group study. BMJ Qual Saf 2012;21(5):361-368. doi:10.1136/bmjqs-2011-000299.

35. Moumtzoglou A. Reporting adverse events: Greek doctor and nurse attitudes. Int J Health Care Qual Assur. 2010;23(7):680687. doi:10.1108/09526861011071607.

36. Hosford SB. The Impact of External Interventions on Improvising the Quality of Patient Safety in Hospitals [dissertation]. Tempe, AZ: University of Phoenix; 2007.

37. Hoover DL. Exploring Military Physician Perceptions of Medical Error [dissertation]. Minneapolis, MN: Capella University; 2007.

38. Walsh K, Antony J. Improving patient safety and quality: what are the challenges and gaps in introducing an integrated electronic adverse incident and recording system within health care industry? Int J Health Care Qual Assur. 2007;20(2-3):107115. doi:10.1108/09526860710731807.

39. Thomas EJ, Petersen LA. Measuring errors and adverse events in health care. J Gen Intern Med. 2003;18(1):61-67. doi:10.1046/ j.1525-1497.2003.20147.x

40. Uribe CL, Schweikhart SB, Pathak DS, Dow M, Marsh GB. Perceived barriers to medical-error reporting: an exploratory investigation. J Healthc Manag. 2002;47(4):263-279. doi:10.1097/00115514-200207000-00009.

41. Harenstam KP, Elg M, Svensson C, Brommels M, Ovretveit J. Patient safety as perceived by Swedish leaders. Int J Health Care Qual Assur. 2009;22(2):168-182. doi:10.1108/09526860910944656. 